First publ. in: Applied Physics / A 70 (2000), 5, pp. 535-539

\title{
The electronic structure and the band gap of nano-sized Si particles: competition between quantum confinemen and surface reconstruction
}

\author{
M. Maus, G. Ganteför, W. Eberhardt \\ Institut für Festkörperforschung, Forschungszentrum Jülich GmbH, 52425 Jülich, Germany
}

\begin{abstract}
The electronic structure and especially the band gap of $\mathrm{Si}_{n}$ clusters ( $n=3-45$ atoms) is studied by photoelectron spectroscopy. Contrary to expectations of quantum conf nement, almost all clusters studied here have a band gap smaller than that of crystalline $\mathrm{Si}$ or even display a continuous (metallic) density of states. We attribute this to covalent bond formation analogous to the reconstructions observed on single-crystal surfaces. Additionally, for $\mathrm{Si}_{30}$ and $\mathrm{Si}_{33}$ a gap size of $0.6 \mathrm{eV}(0.4 \mathrm{eV})$ is observed, supporting the prediction of stable, spherically symmetric structures of these particular clusters.
\end{abstract}

PACS: 71.20.Tx; 73.20.Dx; 36.40.-c

Quantum conf nement in nanostructures fabricated from Si, such as thin $\mathrm{flms}$ or porous $\mathrm{Si}$, results in a renormalization of the band gap [1]. Energy gap values more than triple the size of crystalline Si have been reported for these systems [2,3]. Moreover, the changes in the electronic structure caused by quantum conf nement have also resulted in optical activity of these modif ed structures, a phenomenon very much sought after by developers of optoelectronics but prevented in bulk Si by an indirect band gap.

Calculations for small $\mathrm{Si}$ quantum dots and $\mathrm{Si}$ nanostructures containing typically 1000 atoms are also predicting a considerable opening of the band gap up to an energy of $4 \mathrm{eV}$ [4]. In contrast to this result, cluster calculations of small $\mathrm{Si}$ clusters containing fewer than 10 atoms show that this trend does not continue in the size range of very small particles $[5,6]$. The onset of the calculated absorption spectra of Si clusters containing fewer than 10 atoms is typically at values around $2 \mathrm{eV}$ [6]. For only slightly larger clusters in the size range between 10-20 atoms classical 'force f eld' calculations predict that these clusters are either found in 'metallic' or 'covalent' structures, as characterized by their bond angles [7]. All of these have an energy gap smaller than or of similar size to bulk-crystalline $\mathrm{Si}$. Additionally for some of the $\mathrm{Si}_{n}^{-}$clusters, especially $n=33,39$, and 45 , very interesting spherical-shaped structures have been proposed to exist by calculations [8]. These spherical structures are quite similar to the fullerenes formed by carbon atoms, even though they are not hollow structures. Again, these structures should exhibit a special stability and, in addition to that, largely degenerate electronic states due to their high symmetry.

On the experimental side the picture is similarly confusing. Early photoemission spectra of small $\mathrm{Si}_{n}^{-}$clusters $(n=3-12)$ reveal very broad features with an almost continuous density of states, with the exception of $\mathrm{Si}_{4}^{-}, \mathrm{Si}_{6}^{-}$, and $\mathrm{Si}_{10}^{-}$, which all exhibit a gap of approximately $1 \mathrm{eV}[9,10]$. High-resolution studies, where the vibrational f nestructure was resolved for $\mathrm{Si}_{n}$ clusters for $n=3-7$, confirme these results of the band gap [11]. Furthermore, in an experiment probing the optical absorption of clusters, the quite unexpected result was reported that all $\mathrm{Si}_{n}$ clusters for $n=18-40$ exhibit a very similar, almost featureless absorption spectrum [12]. Recently, mobility measurements have been carried out for Si cluster cations concentrating on the intermediate size range between $n=10$ and 26 and compared with calculations [13]. However, even these careful and extensive studies could not give any def nite answers about the $\mathrm{Si}$ cluster structures in this size range. This leaves $\mathrm{Si}_{7}$ as the largest cluster with a well-conf rmed structure, a pentagonal bi-pyramid, which also seems to persist upon isolation of these clusters in a matrix [14].

\section{Experimental}

In order to investigate the evolution of the band gap and the electronic structure of small $\mathrm{Si}$ clusters in more detail, we here present a photoemission study of small massselected $\mathrm{Si}_{n}^{-}$clusters $(n=3-45)$. The question we hope to answer in these experiments is whether the electronic structure is largely determined by quantum size effects or by the formation of covalent bonds. The $\mathrm{Si}_{n}^{-}$clusters are generated in a PACIS (pulsed arc cluster ion source), which has been optimized for generation of large $\mathrm{Si}_{n}^{-}$clusters $(n=10-100)$ [15]. An effective cooling of the cluster anions turned out to be crucial for the observation of structured spectra. This cooling is achieved using a 0.5 -m-long ex- 
tender with an inner diameter of $2.5 \mathrm{~mm}$. The anions are mass-separated in a time-of-fli ht mass spectrometer and the electron spectroscopy is performed using a "magnetic bottle" time-of-f ight electron spectrometer [16]. The photon energy is $h v=4.66 \mathrm{eV}$, corresponding to the 4th harmonic of a Nd:YAG laser.

The band gap is measured directly using PES of negatively charged clusters $[17,18]$. To f rst order, the photoelectron spectra reveal the density of states of the negatively charged species. In order to establish a procedure relating the energy gap of the neutral species with the observations of features in the photoemission spectra of the anions, one has to turn to a molecular framework. Here the photoemission process is described by transitions from the ground state of the negative ion into the electronic states of the neutral system. The highest energy emission in the photoelectron spectrum of the anion corresponds to a transition into the ground state of the neutral system. Accordingly, the other peaks observed in the photoelectron spectra correspond to transitions into electronically (or vibrationally) excited states of the neutral cluster. The energy separation observed between the highest kinetic energy feature and the next one in the photoemission spectra thus directly and unambiguously ref ects the energy difference between the neutral ground state and the frst excited electronic state. This is also referred to as the HOMOLUMO gap of the neutral cluster.

In the solid-state language the energy separation between the ground state and the frst excited electronic state corresponds to the band gap. In a neutral cluster with an electronic closed shell the additional electron of the anion occupies the conduction band (LUMO) and thus gives rise to a small peak at low binding energy (BE). The emission from the valence band (HOMO) is located at higher BE and the measured energy difference between the small peak and the valence band onset directly corresponds to the band gap. If the neutral cluster does not have a closed shell conf guration, no separated peak should be observed for the anion and the cluster is designated as "metallic".

\section{Results and discussion}

Figure 1 shows a series of photoemission spectra of $\mathrm{Si}_{n}^{-}$ clusters with $n=3-11$. These spectra of $\mathrm{Si}_{3}^{-}-\mathrm{Si}_{11}^{-}$basically agree with the earlier measurements [9-11]. The gap values are derived from these spectra by extrapolation of the high-energy f anks of the photoemission features as indicated by the arrows in Fig. 1. Up to $n=10$ some clusters with intermediate-to-large gaps $(0.9-1.5 \mathrm{eV})$ are observed $\left(\mathrm{Si}_{4}\right.$, $\mathrm{Si}_{6}, \mathrm{Si}_{7}, \mathrm{Si}_{10}$ ), whereas $\mathrm{Si}_{9}$ exhibits a double feature with an energy separation of $0.3 \mathrm{eV}$, but a value for a gap is difficul to deduce. The spectra of $\mathrm{Si}_{3}, \mathrm{Si}_{5}$, and $\mathrm{Si}_{8}$ indicate small gaps of less than $0.5 \mathrm{eV}$. For $\mathrm{Si}_{6}$ we suspect two isomers to be present in the beam with largely different gap values of 0.7 and $0.95 \mathrm{eV}$ as proposed by Binggeli and Chelikovsky [5]. Also for $\mathrm{Si}_{7}$ Neumark [11] reports a smaller gap around $1.1 \mathrm{eV}$ taken from the extrapolation of a low-energy background feature in their spectra. In our spectrum however the second electronic state is observed in total and consequently we derive a larger gap value of $1.5 \mathrm{eV}$ for this cluster.

The photoemission spectra for $\mathrm{Si}_{n}^{-}$clusters for $n=3-20$ are shown in Fig. 2. We have deliberately included the spec-
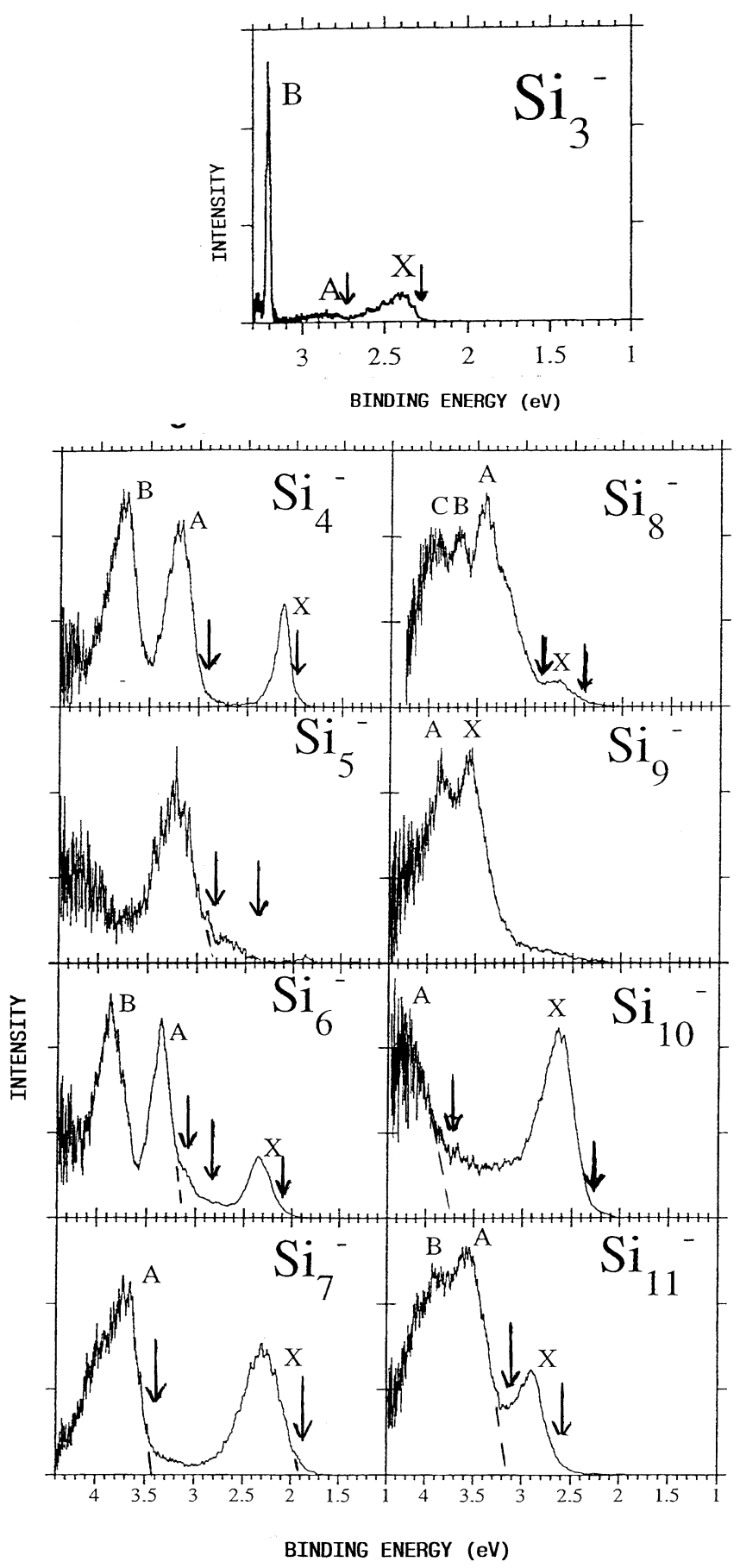

Fig. 1. Photoelectron spectra of $\mathrm{Si}_{n}^{-}$clusters with $n=3-11$ atoms. The photon energy is $h v=4.66 \mathrm{eV}$, except for $\mathrm{Sn}_{3}^{-}$, which is taken with $h v=4.025 \mathrm{eV}$. The arrows mark the extrapolated onsets of the photoemission features used in the determination of the band gaps

tra of the smaller clusters in this f gure in order to facilitate a direct comparison between the smaller and larger clusters. Several spectra in Fig. 2 exhibit a feature at relatively low BE (black), which we assign to the emission from the conduction band of the neutral cluster. Between $\mathrm{Si}_{10}^{-}-\mathrm{Si}_{14}^{-}$the threshold for photoemission of the anion, which approximately corresponds to the electron aff nity of the closed shell neutral species, increases from about $2.5 \mathrm{eV}$ (for $n=3-10$ ) to $3.5 \mathrm{eV}$ for $n=13,14,17$, and 18 . This change is accompanied by 


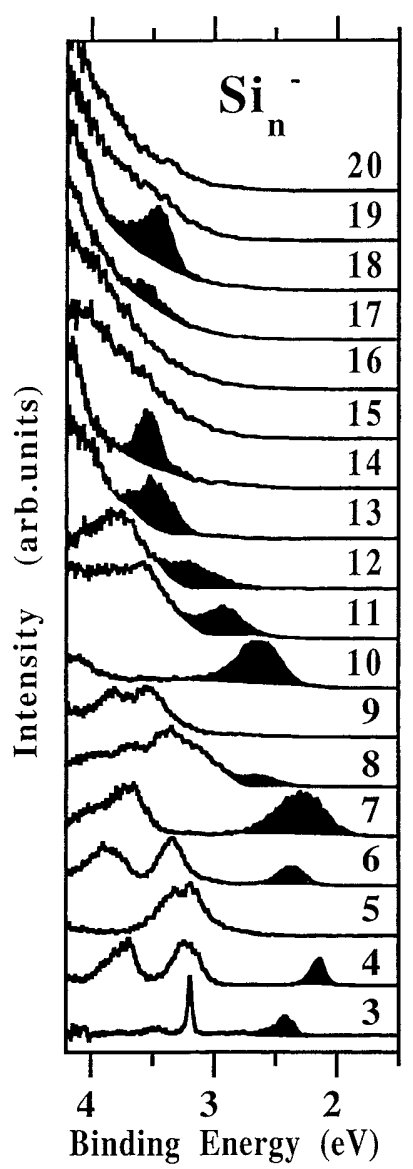

Fig. 2. Photoelectron spectra of $\mathrm{Si}_{n}^{-}$clusters with $n=3-20$ atoms. The photon energy is $h v=4.66 \mathrm{eV}$. The features marked in black are assigned to photoemission from the "conduction" band occupied by the additional electron

an increase of the BE of the valence band emission and a decrease of the average gap size $(n=11-14:<0.5 \mathrm{eV})$. The overall increase of the BEs is observed for clusters of all elements and is generally attributed to an electrostatic effect. A similar trend is observed in the calculated IP of the neutral clusters, averaged over various possible structures, where the IP in the size range $n>12$ is substantially lower than for sizes $n \leq 12[13]$.

As a consequence, for $n>12$ only the upper edge of the valence band is observed under our experimental conditions. For these clusters, the valence band emission gives rise to an emission signal which increases monotonously towards higher $\mathrm{BE}$ (see, for example, the spectra of $\mathrm{Si}_{15}^{-}, \mathrm{Si}_{16}^{-}, \mathrm{Si}_{19}^{-}$, $\mathrm{Si}_{20}^{-}$). If this valence band tail can be observed only, we consider the cluster to be an open-shell "metallic" species. This is the case for $n=15,16$ and almost all clusters larger than $\mathrm{Si}_{18}$. This low-energy tail corresponds to a superposition of the vibrationally broadened edge of the valence band, a thermionic emission signal, and a contribution from inelastically scattered slow electrons [19]. We also have to note here that in investigations of the structures of neutral and cationic clusters in this size range, quite a few clusters were found to exhibit a large structural rearrangement upon ionization [13]. In the photoelectron spectra this also could give rise to indistinguishable features broadened by unresolved vibrational structures.
For the "semi-conducting" clusters, where we could identify the additional electron of the anion occupying the conduction band, a peak appears at low BE additional to the valence band emission $(n=13,14,17,18)$. The gap size cannot be determined accurately, because the exact position of the upper edge of the valence band is unknown (due to the broadening effects and the tail of the thermionic emission). We estimate these gaps to be comparable to the one of $\mathrm{Si}_{12}(0.4 \mathrm{eV})$.

For the larger cluster with $n=19-45$ we found no reproducible features corresponding to the existence of closedshell "magic number" species with the two exceptions $\mathrm{Si}_{30}$ and $\mathrm{Si}_{33}$. In this context it is interesting to note that the structure of $\mathrm{Si}_{9}$ is proposed to constitute a building block for structures of the larger clusters in the size range of $n=20-26$ [13]. Since our experiments show a very small or even vanishing gap for $\mathrm{Si}_{9}$ the larger structures building upon prolate stacks of units of $\mathrm{Si}_{9}$ could be expected to have a small or vanishing gap also.

Figure 3 displays an expanded view of the spectra of the $\mathrm{Si}_{n}^{-}$clusters with $n=30-34$. In these spectra, the monotonously increasing emission signal (see, for example, the spectrum of $\mathrm{Si}_{20}^{-}$in Fig. 2) has been subtracted. This signal is almost independent of size, which indicates that its origin is probably due to thermionic emission and inelastic scattering. The difference spectra display the direct emission features only. However, in most cases the difference spectra look like the ones of $\mathrm{Si}_{31}^{-}, \mathrm{Si}_{32}^{-}$, and $\mathrm{Si}_{34}^{-}$.

The spectrum of $\mathrm{Si}_{30}^{-}$shows a peak centered at $3.45 \mathrm{eV} \mathrm{BE}$. This is approximately the same $\mathrm{BE}$ as for the corresponding feature in the spectrums of $\mathrm{Si}_{18}^{-}(3.4 \mathrm{eV} \mathrm{BE})$. However, the upper edge of the valence band is probably located at a higher $\mathrm{BE}$ compared to $\mathrm{Si}_{18}^{-}$due to the overall increase of the BEs with increasing size. Therefore, the size of the band gap is estimated to be $0.6 \pm 0.2 \mathrm{eV}$. The largest clus-

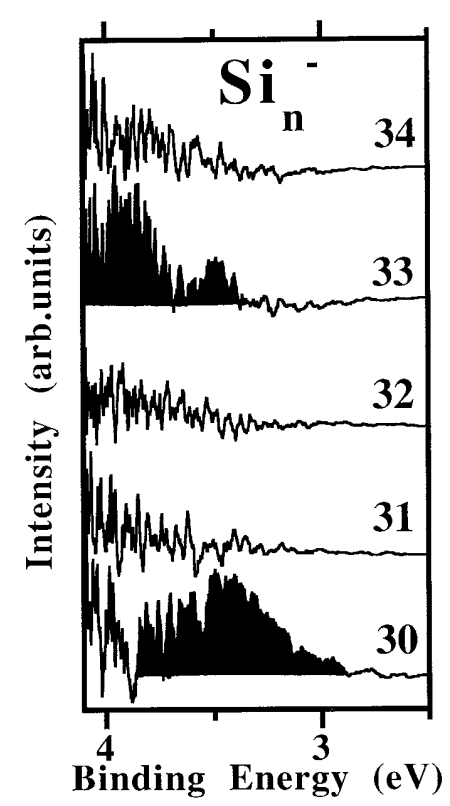

Fig. 3. Expanded view of high binding energy range of photoelectron spectra of $\mathrm{Si}_{n}^{-}$clusters with $n=30-34$ atoms. The photon energy is $h v=4.66 \mathrm{eV}$. The monotonous increasing emission signal (see, for example, the spectrum of $\mathrm{Si}_{20}^{-}$in Fig. 2) has been subtracted. This procedure yields one or two peaks (marked black) for $\mathrm{Si}_{30}^{-}$and $\mathrm{Si}_{33}^{-}$, respectively 
ter, which displays any structure superimposed on the valence band emission is $\mathrm{Si}_{33}^{-}$. The spectrum shows two reproducible structures with a splitting of $0.4 \pm 0.15 \mathrm{eV}$.

These results on $\mathrm{Si}$ clusters are in contrast to our observations of cluster systems consisting of metal atoms reported earlier $[17,18]$. We found in these earlier investigations that, even though some of the electrons clearly exhibit delocalized metallic behavior, small particles containing up to about 40 atoms do exhibit a band gap of at least several tenths of an $\mathrm{eV}$. Thus all these particles are semiconductors, in accordance with the classical definition Moreover, in general terms the electronic structure of these 'metal' clusters is clearly determined by quantum size effects. Already a simple shell model based upon the quantum mechanics of delocalized particles in a single, spherically symmetric potential well describes the electronic structure of these particles quite well. The agreement between theory and experiment is improved considerably, when deviations from the spherical symmetry are taken into account as well $[17,18]$.

Figure 4 shows a summary plot of the values of the band gaps observed in our experiments. Apart from $\mathrm{S}_{7}$ and $\mathrm{Si}_{10}$, all clusters exhibit a band gap smaller than bulk-crystalline $\mathrm{Si}$. For the small clusters $(n<11)$ this is consistent with the theoretical analysis [5] of low-resolution photoelectron spectra [9] of some very small Si clusters, containing 3-7 and 10 atoms. These clusters have no internal atoms and exhibit quite small energy gaps. The electronic structure and the band gap of these small clusters agrees quite well with LDA calculations whereby it turned out to be essential that the structure of the particles was determined by simulated annealing. The high resolution studies of Neumark [11] largely conf rm these conclusions.

In adopting a global perspective originating from a solidstate physics point of view, the small or vanishing gap values of the larger clusters $(11<n<45)$ may be reconciled by taking into account that in this size range most of the atoms are located on the surface of the cluster. Even a cluster with 30 or 45 atoms has more than $80 \%$ of its atoms located at the outer (surface) shell. Presumably, due to covalent bonding and the relaxation of the atoms on the surface of the three-dimensional cluster these atoms rearrange themselves

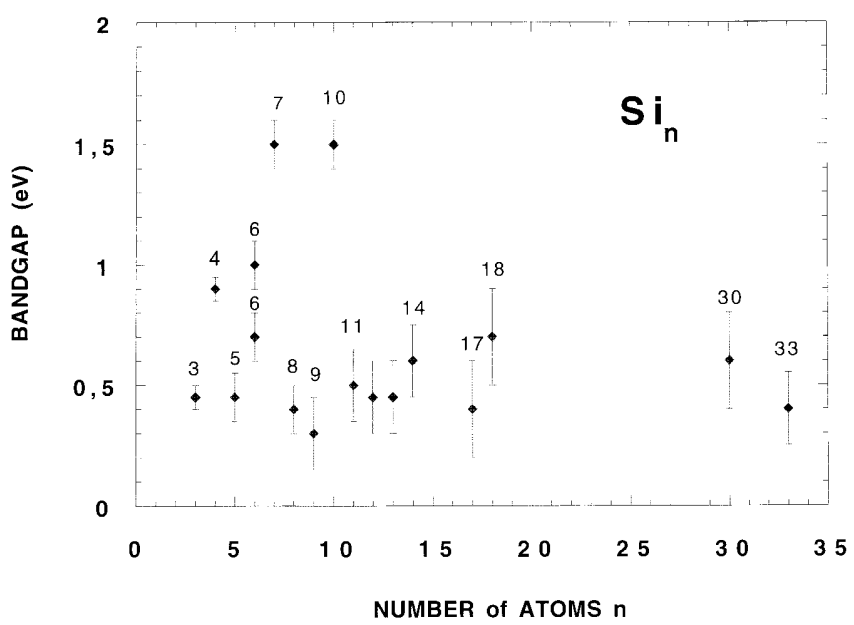

Fig. 4. Band gap of neutral $\mathrm{Si}_{n}$ clusters $(n=4-35)$ estimated from the photoelectron spectra of the anions. For the clusters with $n=19-29$ and $34-45$ our spectra show no indication for the existence of a gap in a similar fashion as on reconstructed single-crystal surfaces [20]. Pair formation results in lowering of the number of dangling bonds and thus reduces the total energy of the cluster. The band gap values of $\mathrm{Si}_{30}$ and $\mathrm{Si}_{33}(0.6 \mathrm{eV}$ and $0.4 \mathrm{eV}$, respectively) are quite similar to the values for reconstructed surfaces.

From Si single-crystal surfaces we know that, for example, dimer formation is observed, lowering the number of dangling bonds and thus the total energy of the surface. As a consequence of these reconstructions the electronic structure of these surfaces has a much smaller energy gap than the bulk material and sometimes, as in the case of Si[111] $7 \times 7$, the surface is even metallic [20]. The $2 \times 1$ reconstruction of the $\mathrm{Si}(111)$ surface, on the other hand, results in a "surface" band gap of $0.4 \mathrm{eV}$ [20]. This corresponds to the splitting of the bonding and antibonding bands of the dimers, which form at the surface in the reconstruction process. According to calculations, the surface atoms of a $\mathrm{Si}_{33}$ cluster also form dimers [8]. A complete saturation of the dangling bonds by dimer formation is only possible for clusters of certain sizes and well-define structures, otherwise unsaturated bonds remain. This model reconciles our observations. It can explain the apparent metallic character of most medium-sized $\mathrm{Si}_{n}$ clusters and also the band gap of $0.4 \mathrm{eV}$ for the magic number cluster $\mathrm{Si}_{33}$ due to the formation of dimers at the cluster surface.

\section{Conclusions}

For $\mathrm{Si}_{n}$ clusters $(3<n<45)$ we $\mathrm{f}$ nd only two clusters, $\mathrm{S}_{7}$ and $\mathrm{Si}_{10}$, with a band gap larger than the one of bulk-crystalline $\mathrm{Si}$, contrary to the expectation based on quantum conf nement. The effect of surface reconstruction and covalent bond formation reducing the number of dangling bonds seems to dominate the quantum size effect. This is not surprising, since almost all $\mathrm{Si}$ atoms in such a small cluster are "surface" atoms. Most of the medium-size clusters $(n=20-45)$ in our study seem to be open-shell "metallic" species, which is also the case for the $7 \times 7$ reconstruction of the $\operatorname{Si}(111)$ surface. For $n>10$, we f nd only a few $\mathrm{Si}_{n}$ clusters with a closed-shell electronic conf guration in the neutral state and a small band gap in the order of $0.4-0.6 \mathrm{eV}\left(\mathrm{Si}_{11}, \mathrm{Si}_{14}, \mathrm{Si}_{17}\right.$, $\mathrm{Si}_{18}, \mathrm{Si}_{30}, \mathrm{Si}_{33}$ ). The detection of a band gap for the two latter clusters $\left(\mathrm{Si}_{30}\right.$ and $\left.\mathrm{Si}_{33}\right)$ could be explained by the high stability spherical-shape structures predicted to exist by theory. Again, since the surface of these spherical clusters exhibits pair formation just as on some single-crystal surfaces, a band gap value of 0.4 to $0.6 \mathrm{eV}$ is not unrealistic for these species.

\section{References}

1. S. Eisebitt, J. Lüning, J.-E. Rubensson, T. van Buuren, S.N. Patitsas, T. Tiedje, M. Berger, R. Arens-Fischer, S. Frohnhoff, W. Eberhardt: Solid State Commun. 97, 549 (1996)

2. T. van Buuren, T. Tiedje, J.R. Dahn, B.M. Way: Appl. Phys. Lett. 63, 2911 (1993)

3. M. Ben-Chorin, B. Averboulch, D. Kovalev, G. Polisski, F. Koch: Phys. Rev. Lett. 77, 763 (1996)

4. C.-Y. Yeh, S.B. Zhang, A. Zunger: Phys. Rev. B 50, 14405 (1994)

5. N. Binggeli, J.R. Chelikowsky: Phys. Rev. Lett. 75, 495 (1995) 
6. A. Rubio, J.A. Alonso, X. Blase, L.C. Balbas, S.G. Louie: Phys. Rev. Lett. 77, 247 (1996)

7. J.R. Chelikowsky, J.C. Phillips: Phys. Rev. Lett. 63, 1653 (1989)

8. U. Röthlisberger, W. Andreoni, M. Parrinello: Phys. Rev. Lett. 72, 665 (1994)

9. O. Cheshnovsky, S.H. Yang, C.L. Pettiette, M.J. Craycraft, Y. Liu, R.E. Smalley: Chem. Phys. Lett. 138, 119 (1987)

10. T.N. Kitsopoulos, C.J. Chick, A. Weaver, D.M. Neumark: J. Chem. Phys. 93, 6108 (1990)

11. C. Xu, T.R. Taylor, G.R. Burton, D.M. Neumark: J. Chem. Phys. 108, 1395 (1998)

12. K.D. Rinnen, M. Mandich: Phys. Rev. Lett. 69, 1823 (1992)

13. K.-M. Ho, A.A. Shvartsburg, B. Pan, Z.-Y. Lu, C.-Z. Wang, J.G. Wacker, J.L. Fai, M.F. Jarrold: Nature 392， 582 (1998); B. Liu, Z.-Y. Lu, B. Pan, C.-Z. Wang, K.-M. Ho, A.A. Shvartsburg, M.F. Jarrold: J. Chem. Phys. 109, 9401 (1998)
14. E.C. Honea, A. Ogura, D.R. Peale, C. Felix, C.A. Murray, K. Raghavachari, W.O. Sprenger, M.F. Jarrold, W.L. Brown: J. Chem. Phys. 110, 12161 (1999)

15. C.-Y. Cha, G. Ganteför, W. Eberhardt: Rev. Sei. Instrum. 63, 5661 (1992)

16. H. Handschuh, G. Ganteför, W. Eberhardt: Rev. Sei. Instrum. 66, 3838 (1995)

17. G. Ganteför, C.-Y. Cha, H. Handschuh, G. Schulze Icking-Konert, B. Kessler, O. Gunnarsson, W. Eberhardt: J. Electr. Spectrosc. 76, 37 (1995)

18. H. Handschuh, C.-Y. Cha, H. Möller, P.S. Bechthold, G. Ganteför, W. Eberhardt: Chem. Phys. Lett. 227, 496 (1994)

19. G. Ganteför, W. Eberhardt, H. Weidele, D. Kreisle, E. Recknagel: Phys. Rev. Lett. 77, 4524 (1996)

20. W. Mönch: Semiconductor Surfaces and Interfaces, Springer Series in Surface Science, Vol. 26 (Springer, Berlin, Heidelberg 1993) 